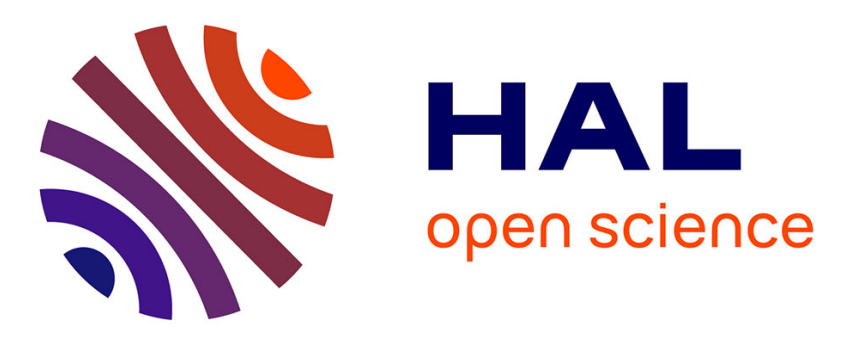

\title{
First experiments on MagPieR: a planar wireless magnetic and piezoelectric microrobot.
}

Ioan Alexandru Ivan, Gilgueng Hwang, Joël Agnus, Micky Rakotondrabe, Nicolas Chaillet, Stéphane Régnier

\section{- To cite this version:}

Ioan Alexandru Ivan, Gilgueng Hwang, Joël Agnus, Micky Rakotondrabe, Nicolas Chaillet, et al.. First experiments on MagPieR: a planar wireless magnetic and piezoelectric microrobot.. IEEE International Conference on Robotics and Automation, ICRA'11., May 2011, Shanghaï, China. pp.102-108. hal-00797894

\section{HAL Id: hal-00797894 \\ https://hal.science/hal-00797894}

Submitted on 7 Mar 2013

HAL is a multi-disciplinary open access archive for the deposit and dissemination of scientific research documents, whether they are published or not. The documents may come from teaching and research institutions in France or abroad, or from public or private research centers.
L'archive ouverte pluridisciplinaire HAL, est destinée au dépôt et à la diffusion de documents scientifiques de niveau recherche, publiés ou non, émanant des établissements d'enseignement et de recherche français ou étrangers, des laboratoires publics ou privés. 


\title{
First experiments on MagPieR: a planar wireless magnetic and piezoelectric microrobot
}

\author{
Ioan Alexandru Ivan, Member, IEEE, Gilgueng Hwang, Member, IEEE, Joel Agnus, Micky \\ Rakotondrabe, Member, IEEE, Nicolas Chaillet, Member, IEEE, Stéphane Régnier, Member, IEEE
}

\begin{abstract}
The paper documents the principle and experiments of the "2mm dash" winner at NIST IEEE Mobile Microrobotics Challenge held at ICRA2010 in Alaska [1]. Submission is made for the special session "ICRA Robot Challenge: Advancing Research Through Competitions".

The new MagPieR microrobot was specially designed for breaking the speed record, providing a planar magnetic actuation with an optimised coils setup and a subsequent piezoelectric actuation for improved sliding condition. The paper describes the principle of actuation, the microrobot manufacturing flowchart and the assembly setup. Some simulations are provided with a first series of experimental data and conclusions.
\end{abstract}

\section{INTRODUCTION}

$\mathrm{R}$ ecent progress in micro-/nanotechnologies enabled micromechanical systems (MEMS) sensors and actuators. A new emerging field has recently appeared in the micro and nanotechnologies, the untethered, submillimeter, micro or nano-sized robots [2]. They present potential applications in biomedical diagnosis, targeted drug delivery or microassembly. Many issues including system design, microfabrication, remote power sourcing and control must still be considered and developed towards real applications.

Micron-size robots can also solve one of the most important problems of the micromanipulation at this scale. Their abilities of manipulating micron scale objects are very promising but challenging. Untethered microrobot approaches offer a valuable alternative to conventional micro-robotic systems based on tethered manipulation systems. They are not kinematically constrained, thus they allow a larger manipulation workspace, access to unstructured environment and even liquid environments in which can be immerged for avoiding adhesion or sticking effects.

Manuscript received September 15, 2010. This work was supported in part by the EU FP7SP3-People Program under Grant No: PIEF-GA-2008-219412 (NewMicro-Robotic Systems featuring Piezoelectric Adaptive MicroStructures for Sensing and Actuating, with Associated Embedded Control: MicroPAdS).

Ioan Alexandru Ivan, Micky Rakotondrabe, Joel Agnus and Nicolas Chaillet are with FEMTO-st Institute, UMR CNRS-6174 / UFC / ENSMM / UTBM Automatic Control and Micro-Mechatronic Systems department (AS2M department) 25000 Besancon - FRANCE (phone: +33-381-402-789; fax: $\quad$ +33-381-402-809; e-mails: alex.ivan@ femto-st.fr, joel.agnus@ens2m.fr, mrakoton@femto-st.fr, nicolas.chaillet@femto-st.fr).

Gilgueng Hwang and Stephane Regnier are with University Pierre et Marie Curie, Institut des Systèmes Intelligents et de Robotique Paris, FRANCE (e-mails: hwang@ @isir.upmc.fr, stephane.regnier@upmc.fr).
The major current challenges consist in innovating efficient remote power sourcing and proper locomotion mechanisms. Drive and propelling such tiny devices require overcoming the nonlinear physics at this scale. Then, the ability to accurately maneuver them represents the next challenge. Current wireless mobile micro-robots have been designed with the power source from electrostatic, electromagnetic, thermal and electrokinetic force (e.g. bacteria propelled systems). For the biological approaches, harnessing biological organisms were demonstrated to give the power to propulsion [3-6]. These have a great potential due to the fact that the energy can be harvested from everywhere through chemical reactions abundant in the environment. However controlling their propulsion is very challenging. As with inorganic approaches, an electrostatic scratch drive actuator-based microrobot was shown in [7]. It has the advantage of using the most dominating controllable force type in the micro-scale. However, its application range is limited due to the need for a specific patterned substrates and high electrical potentials. Other energy conversion mechanisms such as thermal actuators [8] and piezoactuators [9] were demonstrated. Although these principles are well developed and are supported by many physics works, they cannot meet the requirements of the fully untethered microrobots. Among all these approaches, the electromagnetic forces still remain the most reasonable power sources for building untethered microrobots. Electromagnetic stick-slip force was used to drive micron size robots in [10]. Resonating magnetic field was utilized to actuate small mass-spring microrobots in a linear motion [11]. Artificial bacteria were actuated by external rotating magnetic field [12] and electroosmotic force [13]. Multi-DOF electromagnetic levitation force principle was successfully presented in [14].

Although such a dramatic advancement in powering remote propulsion, the range of applications remains still limited. It is mainly due to the lack of functions to be applied to any complex tasks. Real breakthrough must overcome the current maneuvering ability limits by combining the available technologies. Another challenge in micro-robotics is the control of multiple untethered robots for conducting collaborative manipulation tasks. Control of decoupled motion, strategies of manipulation, multirobot cooperation [15][16] are on-going projects and the first results show promising results. Therefore, untethered microrobots with multiple functions could open a new era. Our approach is to 
develop a new hybrid piezo-magnetic powered microrobot to demonstrate a higher planar propulsion performance.

In 2007 NIST launched a competition, the NIST Mobile Nicrorobotics Challenge. Since 2010, this challenge got IEEE support and will occur during the ICRA Conference. A French team, composed by the two institutes FEMTO-ST and ISIR, decided to participate in 2010 to this competition, aiming for the first participation an as fast as possible " $2 \mathrm{~mm}$ dash race". In the $2 \mathrm{~mm}$ dash race [1], a microrobot smaller than $600 \mu \mathrm{m}$ in its largest dimension must cross a distance of two millimeters in a time as short as possible.

A new microrobot called MagPieR (Magnetic Piezoelectric microrobot) was designed for this challenge. The MagPieR mobile microrobot is composed of two heterostructure layers: ferromagnetic material (Nickel) layer for magnetic driving on top of a bulk piezoelectric material (PMN-PT). The microrobot is intended to overcome the surface friction. It can move on the horizontal plane of a capacitor whose bottom electrode is the arena substrate itself and the top electrode is optically transparent conductive glass (ITO glass). A series of high voltage periodic pulses provide out-of-plane piezoelectric strain, making the robot loose adhesion contact with the arena surface for short amounts of time. Subsequently, fast in-plane motions (translation and steering) are insured by external magnetic field gradients provided by closely coupled four electromagnetic coil sets. With this proposed design, the French team won the competition with an RMS (root mean squared) score of 32 ms. The article will describe in details the overall structure of the robot and characterize in a first instance its linear propulsion performance.

The first part of this article deals with the description of the principle drive mechanisms of the microrobot, from process flowchart to overall system implementation. Then, a modeling section will follow with finite elements simulations. Finally the experimental results on a couple microrobot types are shown to reveal the propulsion characteristics such as linearity and the performances. Finally the paper discusses conclusions and further improvements.

\section{SYSTEM OVERVIEW}

\section{A. Operating principle}

The untethered MagPieR microrobot successfully competed to the ICRA 2010 Microrobotic challenge [1]. Given our first participation to this challenge we mainly focused on the $2 \mathrm{~mm}$ dash. In the attempt to provide faster actuation, we chose to combine a new mix of simultaneously piezoelectric and a magnetic type of actuation.

As well known, adhesion forces at microscale become predominant. A microrobot whose size is imposed to less than a half-millimeter will stay "sticked" to its contact surface regardless its inclination. One of the first experiments was investigating the possibility of reducing the adhesion effects by getting vibrating pieces of PZT and PMN-PT piezoelectric material. In Fig.1 we inserted a piece of piezoelectric material between a silicon and a bulk aluminium plate that were inclined at $30 \mathrm{deg}$.. Adjusting the gap to a slightly superior value than to the piezo material thickness $(200 \mu \mathrm{m})$ and upon applying high voltage square impulses $(300 \mathrm{~V}, 100 \mathrm{~Hz})$ and paying attention to the limit of the Paschen curve, we eventually noticed the piezoelectric material loosing its adhesion and starting to slide according to the gravitational laws.

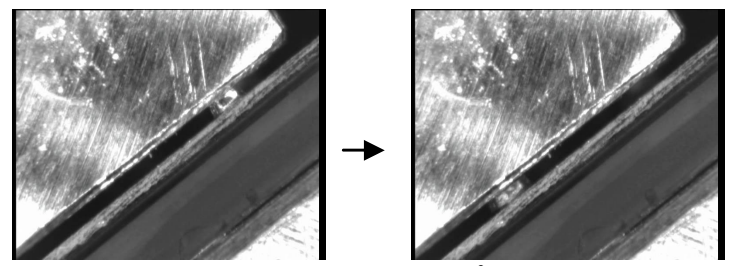

Fig.1 Early experiments on a 500x500x200 $\mu^{3}$ piece of PMN-PT material validated sliding conditions upon applying step impulses of $(0 \ldots+300) \mathrm{V}$ at $100 \mathrm{~Hz}$. The frames are taken at $\sim 100 \mathrm{~ms}$ interval.

Following the positive results in limiting adhesion forces upon vibrating a piezoelectric element, we considered the hybrid actuation principle from Fig.2, which combines piezoelectric out-of-plane vibrations for improved friction-free conditions and magnetic field gradient actuation for in-plane displacement (x-y) and orientation $(\theta)$. For that purpose, the final microrobot was designed of two distinctive layers: a lower bulk piezoelectric layer and a ferromagnetic patterned layer (Fig.4).

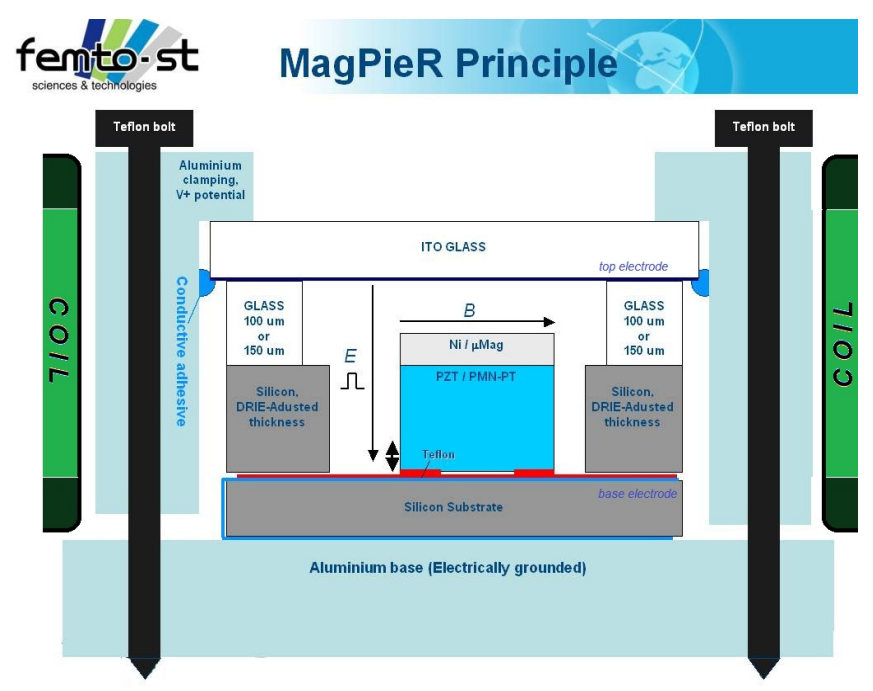

Fig.2 MagPieR Microrobot Principle of actuation and arena schematic.

The microrobot was positioned into a $3.5 \times 2.0 \mathrm{~mm}^{2}$ "challenge arena" whose dimensions were imposed by the competition organizers [1]. In order to be able to visualize the scene perpendicularly, an ITO conductive glass was considered for the top electrode (Fig. 2). Further details about the arena setup are provided in Section-II.D. Details about the coils setup and driving electronics are presented in Section-II.B and in Section-III. 


\section{B. Electronic driving circuit}

The electronic circuit was custom-made, microcontroller-based and embedded. For the " $2 \mathrm{~mm}$ dash" a position feed-back control was not necessary, although after the competition we started studying a couple vision-based approaches.

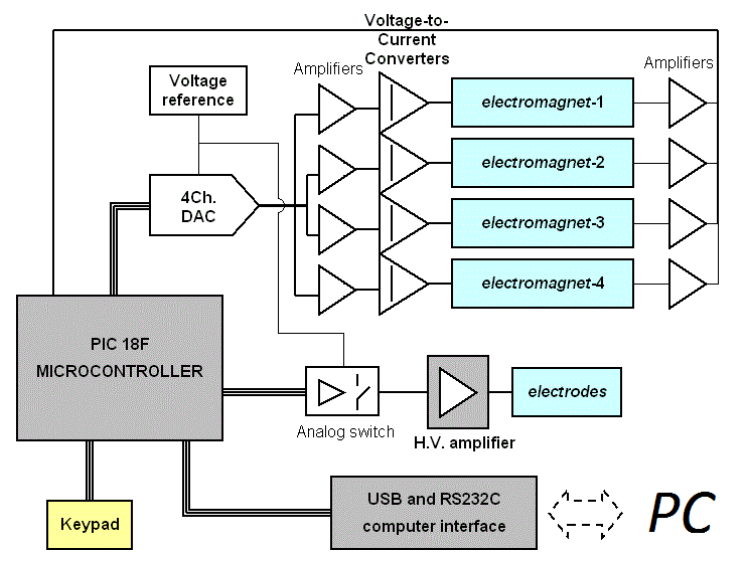

Fig.3 Block diagram of embedded electronic circuit

Once configured through a USB or serial interface (current values, high voltage, impulse times) the system is able to operate without computer connection. We designed high-power op-amp based voltage-to-current converters able to continuously operate up to $1 \mathrm{~A}$ and in impulses up to $4 \mathrm{~A}$. Attention was paid to the coils overheating protection, a feedback voltage intended for the coils temperature evaluation was put in place. However, given optimized coils setup (1300 turns, $17 \mathrm{~mm}$ long, $4 \mathrm{~mm}$ diameter ferromagnetic core), currents of $\leq 1 \mathrm{~A}$ were largely sufficient for the application. An analog switch controlled the high voltage amplifier type KH7602 was connected to the electrodes, in order to activate the piezoelectric effect. A four-directions keypad with some extra buttons (reset, high speed command) served to manually control the microrobot back and forth in any directions, to position it close to the starting line and to launch the $2 \mathrm{~mm}$ dash.

\section{Microrobot design and process flow}

The final microrobot structure consisted of a top Ni layer electrodeposited on a PMN-PT substrate. Piezo PMN-PT material was preferred to the classical PZT ceramic due to the very high longitudinal $d_{33}$ piezoelectric coefficient (3100 $\mathrm{pC} / \mathrm{N}$ compared to $590 \mathrm{pC} / \mathrm{N})$ that finally allowed a significantly reduced voltage to get the same strain (vibration) conditions.

Microrobot flowchart consisted of the following stages:

- Cutting PMN-PT substrate into half-a- $\mathrm{cm}^{2}$ plates;

- Sputtering a $\mathrm{Cu}-\mathrm{Cr}$ layer on the top side;

- Sputtering a Ti-Cr layer on the bottom side;

- Spin coating teflon-based resin on the bottom (optional);

- Ni electroplating of top electrode;

- Saw dicing into small square and rectangular samples;

- Saw trenching the top Ni layer (optional).
The final structures were $224 \mu \mathrm{m}$ thick; of which $200 \mu \mathrm{m}$ the PMN-PT layer and $24 \mu \mathrm{m}$ the Ni layer, as in Fig 4. We used two different geometries, square and rectangular ones as summarized in the Table1.

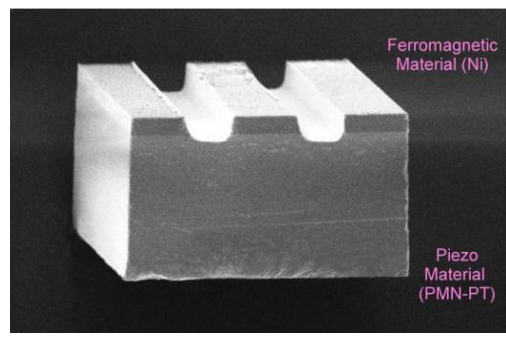

Fig.4 SEM of a MagPier microrobot type I.2 (trenches are $50 \mu \mathrm{m}$ wide).

Table I. MagPieR types used for experiments. Type II.1 competed.

\begin{tabular}{|l|l|l|l|l|}
\hline Type & $\begin{array}{l}\text { Length } \\
{[\boldsymbol{\mu m}]}\end{array}$ & $\begin{array}{l}\text { Width } \\
{[\boldsymbol{\mu m}]}\end{array}$ & $\begin{array}{l}\text { Trench } \\
\text { width }[\boldsymbol{\mu m}]\end{array}$ & $\begin{array}{l}\text { 2mm dash score (ms) } \\
\text { and observations }\end{array}$ \\
\hline T I.1 & 388 & 388 & $50(\mathrm{two})$ & $25 \mathrm{~ms}$ \\
\hline T I.2 & 388 & 388 & 0 & not tested, unstable geom. \\
\hline T II.1 & 388 & 300 & $50($ one $)$ & $15 \mathrm{~ms}$, competition type \\
\hline T II.2 & 388 & 300 & 0 & $9 \mathrm{~ms}$, but unstable \\
\hline
\end{tabular}

Results will be presented more detailed in Section-IV. The models I.1 and II.1 were considered from the beginning with two and one trench, respectively (Fig. 5). That feature allowed a faster and more oscillatory-free orientation along magnetic field lines. We equally tested bulk samples type II.2 (without trenches) like in Fig.11 which naturally showed fastest speeds due to the increased amount of $\mathrm{Ni}$, but they performed in a more unstable manner and we didn't compete with.
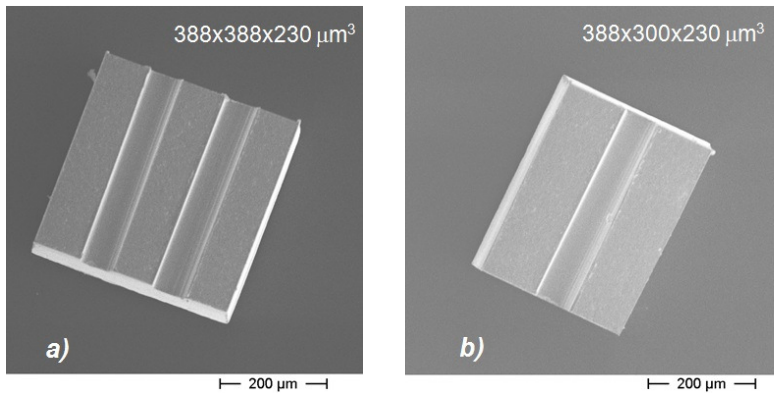

Fig.5 SEM of a MagPier microrobots a) type I.1 and b) type II.1

\section{Arena microfabrication and assembly}

The rules for arena dimensions and tolerances were provided by NIST organizers [1], each team feeling free to use its own materials. We chose a dry environment made of four distinctive layers of glass and silicon (Fig.2) cut at convenient dimensions for a rapid assembly. Figures 6 and 7 illustrate how the robot is encapsulated in the arena assembly by means of two specially designed teflon flanges. Once assembled, preferably in a clean room environment, the microrobot setup can be furthermore attached and detached from the coils base (Fig 7.d). 


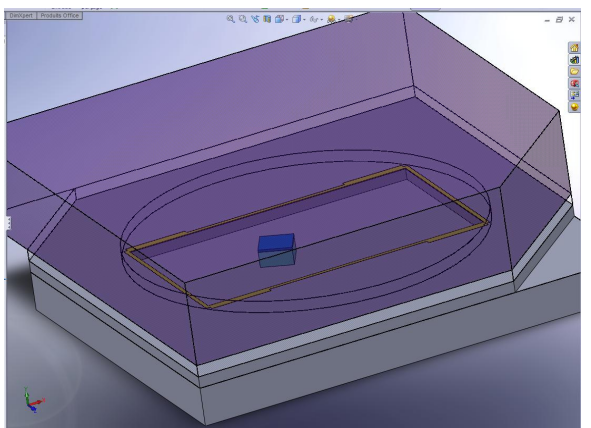

Fig.6 Schematic of the packaged arena consisting of the following layers (from up to bottom): ITO top electrode, glass spacer, silicon border, silicon base.

The four arena layers were $6.5 \times 6.5 \mathrm{~mm}^{2}$ in the lateral size but cut in a manner that allowed the electrical access to the electrodes. The layers are as follows:

1. A top conductive ITO glass acting as transparent top electrode and glued to a wire (up-left in fig.7.c).

2. An intermediate glass layer of $150 \mu \mathrm{m}$ that was cut annularly using an ultrasound machine. This layer served as the dielectric separation.

3. An intermediate silicon layer of $110 \mu \mathrm{m}$ that was microfabricated at both sides using metal sputtering, photolithography and DRIE (deep reactive ion etching) techniques. A thin aluminium layer was used as DRIE mask while a gold film to trace the marks. This layer insures the borders and the marks of the virtual starting and finish lines.

4. A bottom piece cut from a regular silicon layer whose surface was metallized by $\mathrm{Cr}-\mathrm{Al}$ sputtering. This layer served as the base and as the ground electrode, a wire being bonded accordingly.
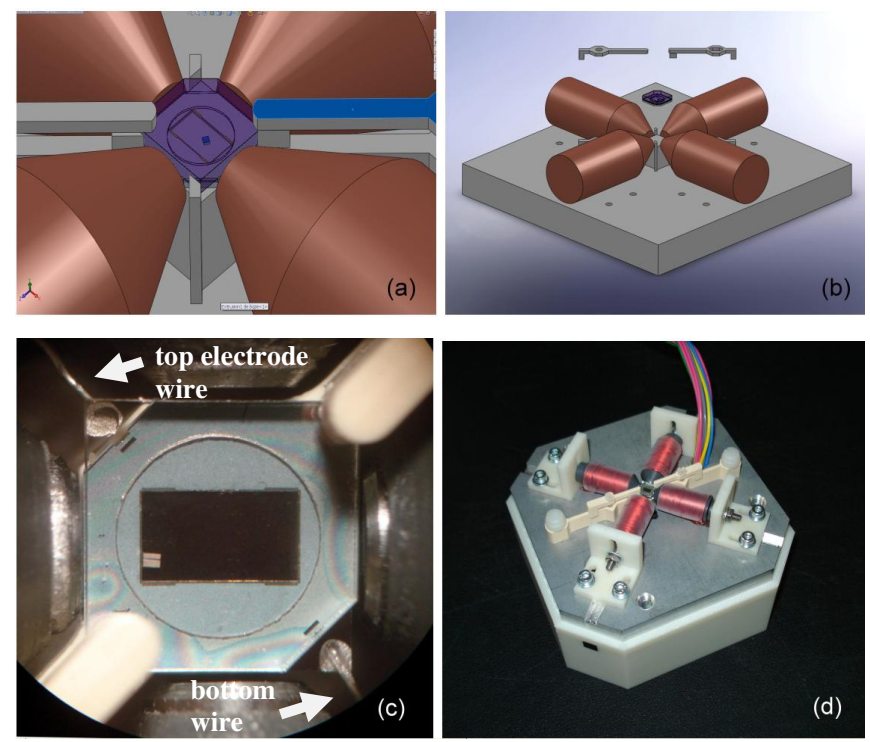

Fig.7 a,b) CAD of the arena assembly, c,d) photos of the arena and of the complete system. Total system size is $11 \times 11 \mathrm{~cm}^{2}$.

\section{Magnetic Field Modelling}

\section{A. Equation of the motion}

Three natures of forces act on the microrobot: mechanical (from friction and adhesions between the robot and the base), electrical (from the electrical field) and magnetic (from the electromagnets) forces. Hence:

$m \vec{\Gamma}=\vec{F}_{\text {mec }}+\vec{F}(\vec{E}, \vec{B})$

where $\vec{\Gamma}$ is the acceleration of the microrobot and $m$ is its mass. $\vec{F}(\vec{E}, \vec{B})$ includes the magnetic force acting in the volume, the piezoelectric stress and the eventual Lorentz force w.r.t. the trajectory .

Since the paper is particularly dedicated for the analysis of performances upon executing the " $2 \mathrm{~mm}$ dash" as required in [1], we assume that:

- for large magnetic field values the contribution of piezoelectric effect is less predominant. Therefore for $2 \mathrm{~mm}$ task we assume that electrical field is null, only one electromagnet is used to actuate the robot. The magnetic force is dependant on the volume $V$ of the ferromagnetic layer and proportional to the gradient of the magnetic field at its location. We assume that during the challenge the axis of the microrobot is very closed to the symmetry axis denoted $x$ of the electromagnet,

- the tangential part of the mechanical force comes from friction during the motion. The normal forces from the weight and from adhesion forces [17] - are compensated by the reaction of the base.

thus:

$m \vec{\Gamma}=\left(\begin{array}{l}-\mu\left(m g+F_{a d h}\right) \\ 0 \\ 0\end{array}\right)+V(\vec{M} \cdot \nabla) \vec{B}$

where $g \cong 10\left[\frac{m}{s^{2}}\right]$ and where the adhesion forces $F_{a d h}$ can be approximated by using plane-to-plane contact in dry media condition (see for example [17]). There are a lot of approaches to model the friction coefficient $\mu$. The choice mainly depends on the conditions and on the expected applications. At the microscale where unwanted stick-slip phenomena often happens during the motion, the elasto-plastic friction model can be efficiently used [18], for a purpose of modelling and control.

\section{B. Magnetic force analysis}

The magnetic force $\vec{F}_{m a g}=V(\vec{M} \cdot \nabla) \vec{B}$, where the magnetization $\vec{M}$ of the robot can be assumed uniform, is volumetric and therefore the required field and field gradient to exert a certain force increases rapidly with the decrease of the sizes of the robot [19].

To estimate and analyze the magnetic field density, we use finite-element-modelling (FEM) software from Comsol Multiphysics. Because of the symmetry geometry of the electromagnet (described in Section II.B), of the negligible robot sizes relative to it and their small separating distances, we decide to employ 2D-axisymmetrical analysis in the FEM software (Fig.8). This allows an ease of computation during 
the processing task. In the software, the coil is represented by a cylindrical (copper) block (Fig.8.a). So, to introduce the electrical excitation, we apply a current density $J$. This density can be computed from the current $i$ that we want to apply to the coil such that $J=\frac{4 i}{\pi d_{w}{ }^{2}}$, where $d_{w}$ is the diameter of the used copper wire. In this analysis we consider $i=0.2 A, 0.5 A$ and $1.0 A$.

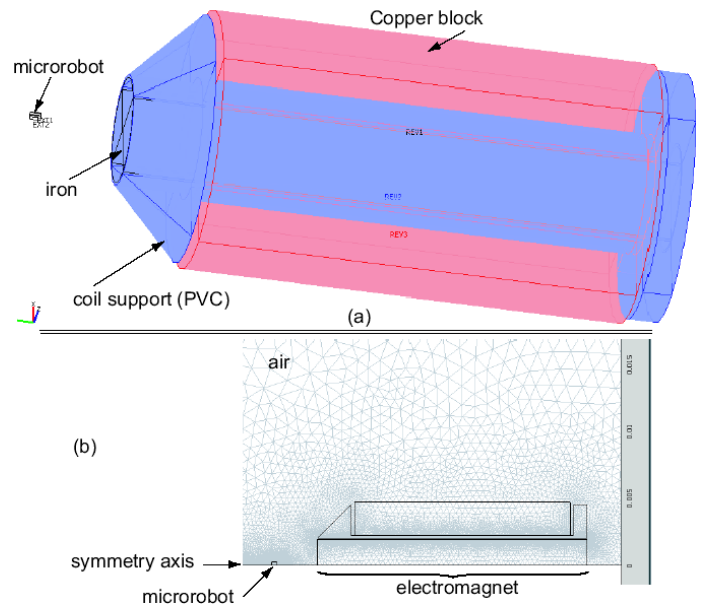

Fig.8: Comsol Multiphysics - FEM analysis. (a): scheme showing the geometrical characteristics and distance between the microrobot and the electromagnet. (b): 2D-axisymmetrical meshing.

The streamline of the magnetic field w.r.t. current density appears as in Fig.9. As we can see, the lines can be assumed to be parallel within the dimensions of the microrobot. This is due to small sizes of the robot and the fact that it is very closed to the symmetry axis $x$ during its motion. Under this configuration, with the electromagnets in close vicinity to the arena, we gain in field gradients but we loose in the orthogonality of the field. The trade-off consists in increasing the distance between coils with the cost of a lower dragging force. The setup allows the mechanical re-configuration of the coils position, as noticeable in Fig 7.d. Research on this topic will be published on a separate paper regarding microrobot in-plane control.

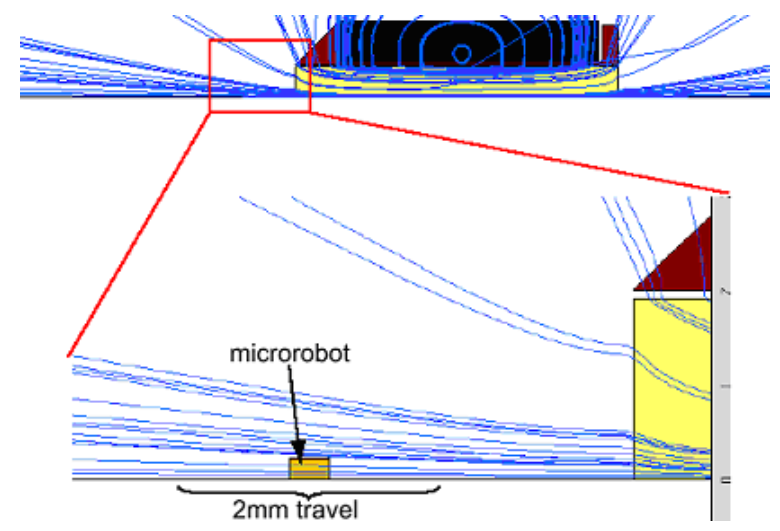

Fig.9 Streamline of the magnetic field.

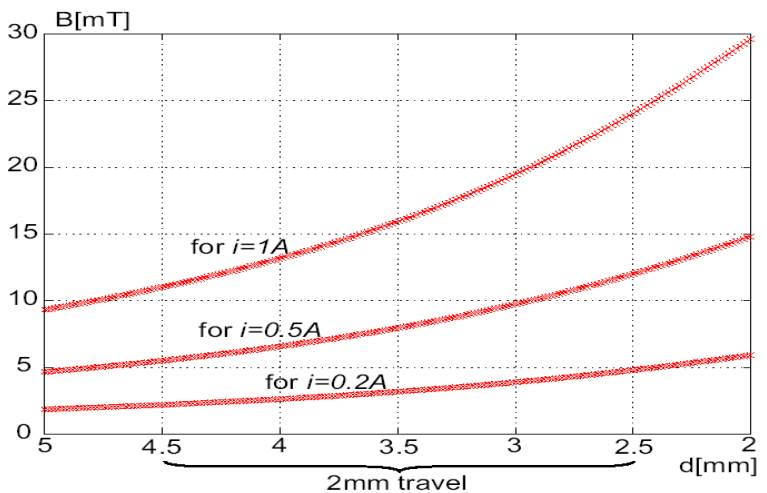

Fig.10 Magnetic field along the distance travelled by the microrobot, during the $2 \mathrm{~mm}$ dash challenge.

Fig.10 shows the simulation results of the magnetic field along $3 \mathrm{~mm}$, which includes the $2 \mathrm{~mm}$ challenge dash (coils in close vicinity to the arena). The field and its gradient increase in an exponential manner as the microrobot moves towards the electromagnet (increasing acceleration).

\section{EXPERIMENTS}

In this section, we demonstrate a few experiments of MagPieR, in order to characterize the propulsion performance and prove eventually the design efficiency. The aim of this section is to analyze the motion of MagPieR in the $2 \mathrm{~mm}$ dash task and try to further improve its design.

\section{A. Propulsion in $2 \mathrm{~mm}$ dash task}

First of all, we describe briefly the $2 \mathrm{~mm}$ dash task demonstrated in the section. The task is to measure the travel time from start to complete stop by a highspeed camera analysis during the MagPieR motion from start to goal line. The surface friction are assumed to be constant. Then two more issues should be considered to accomplish the task. Firstly, a straight linear propulsion increase the energy transfer efficiency of the microrobot. Furthermore, the stopping at the closest point from the goal line should also be important to avoid any redundant motion which still is counted in the travel time. The assembled arena with two different types of MagPieRs were used for the $2 \mathrm{~mm}$ dash task experiments. The specifications of four types of MagPieR and minimum corresponding scores are summarized in Table-I. We chose to compare the fastest types II.1 and II.2 that have similar geometries except one has a trench crossing the longitudinal axis on the ferromagnetic nickel layer while the other present a smooth surface without the trench. Then we aim to reveal the effect of this trench correlated to the propulsion linearity and the performance, thus can validate the proposed design. For a better comparison in propulsion linearity, a high-speed camera with 1000 frames per second (fps) was used to record the videos during the tasks. 

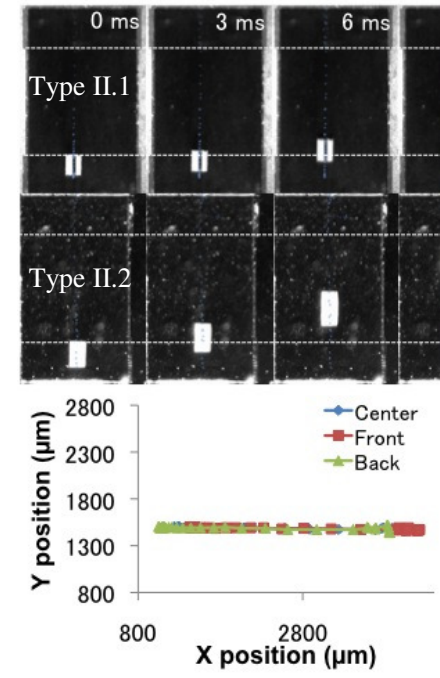

Fig. 11 The captured motions during $2 \mathrm{~mm}$ dash task and their tracked trajectory plots for the two different types of MagPieR. Type II. 1 is shown in the upper part and type II.2 in the lower part. Current imposed value was 1.2A. Aligning motion in the type II. 1 and unstable motion in the type II. 2 were observed (the scale bar is $500 \mu \mathrm{m}$ ).

Figure 11 shows a series of captured images at $3 \mathrm{~ms}$ interval, captured from the original movie taken at $1000 \mathrm{fps}$. As shown in the Figure 11, while the MagPieR type II.1 revealed a propulsion linearity, the type II.2 showed a faster motion. The type II.1 with a trench on top of the ferromagnetic layer took longer time for alignment during their propulsion. It should be noted here that the motion of MagPieR was demonstrated in four different distinctive steps. Firstly, it aligns through the electromagnetic field gradient, continues the linear propulsion then passes the goal line, collides to the wall behind the goal line and finally stops. It implies that we can further improve the propulsion performance either by modifying the input pulse time and controlling the propulsion linearity from design parameters.

Figure 11 also depicts the tracked trajectories of the propulsions of two different types. As shown in the planar trajectory plots, the type II.2 (right plot) loses the propulsion stability by rotating right after the collision to the wall. The travel time estimation in $2 \mathrm{~mm}$ dash task measures the time between the start line and a complete stop behind the goal line. Therefore unnecessary motions can be avoided to further improve the record by about $25-30 \%$.

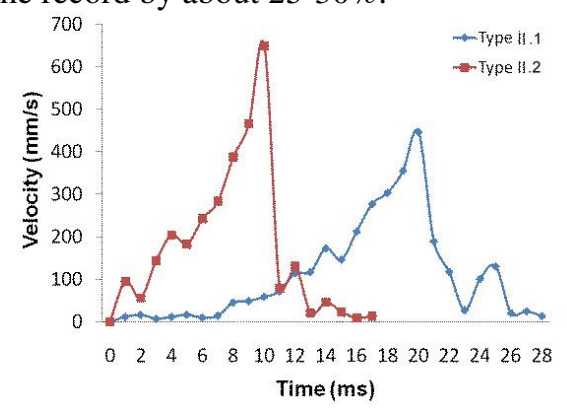

Fig. 12 Velocity characterizations of MagPieR type II.1 and II.2

\section{B. Propulsion performance characterizations}

We further compare the propulsion performance between two different types of MagPieRs. As summarised in the Table I, the type II.1 measures the average record at $14.8 \mathrm{~ms}$ from four different trials (the standard deviation is $4.2 \mathrm{~ms}$ ). And the type II. 2 took average $9.4 \mathrm{~ms}$ with $2 \mathrm{~ms}$ standard deviation. It should be noted that pure travel time between start and goal lines was measured to exclude the effect from collision to wall. Although the propulsion linearity is assured better in the type II. 1 by the better alignment of the trench to the external magnetic field, such deviation of measured time in the type II.1 is attributed to the initial alignment condition. When the trench is initially well aligned through the field, additional motion required for the alignment (a damped oscillatory rotation) that adds friction can be avoided, thus relatively fast enough propulsion can be achieved. Moreover the etched away ferromagnetic layer in the type II.1 reduces the magnetically driven propulsion force. Assuming that the overall inertial effect from slight mass changes is ignorable, the magnetic propulsion force (Eq. 2 in the modeling section) is volumetric thus the type II. 1 loses $49 \%$ of the driving force compared to the type II.2. This can be confirmed from the consistency to the reduction of maximum acceleration in the type II. 1 which is $49.7 \%$.

For more accurate analyses of their propulsion, the velocity and the acceleration curves are displayed on fig 12 . The rapid drop of each curve clearly shows when the MagPieRs were collided to the wall behind the goal line. Therefore it is inevitable to find the optimal input impulse parameter to properly make the MagPieRs stop at the closest to the goal line without any collision.

\section{Improvement of the propulsion}

To avoid the collision to the wall and make the MagPieR stop at the closest distance from the goal line, we aim to find the optimal input step pulse. For this purpose, we have characterized the travel distances of the type II. 1 at each input impulse time in the range of 4 and $223 \mathrm{~ms}$. The measured result is displayed in the Figure 13. As a result, a $14 \mathrm{~ms}$ of input impulse time stopps MagPieR at the closest to the goal line. This parameter could reduce the travel time by avoiding the redundant travel behind the goal line from collision and bouncing. Further surface optimization can even enhance the current propulsion performance.

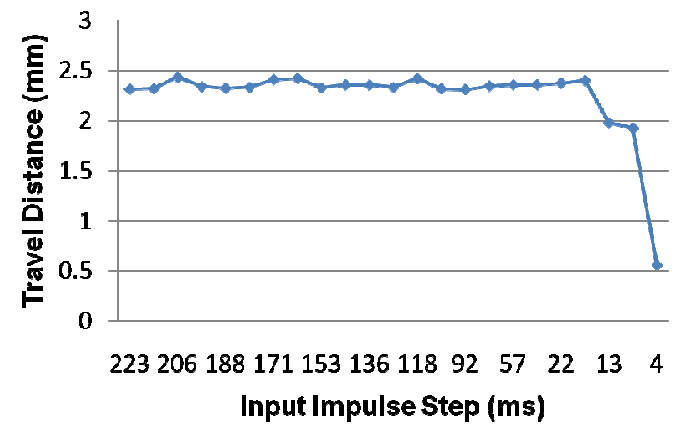

Fig. 13 Propulsion characteristics of stopping distances depending on the input pulse steps. MagPieR type II.1. 


\section{CONCLUSION, CURRENT AND FUtURE WORKS}

We proposed a new microrobot system by combining external piezoelectric and electromagnetic forces. The principle has the advantage of partly overcoming the surface adhesion, allowing faster planar propulsion especially under low field gradients. The microrobot system parts were fabricated using MEMS process and assembled in the clean room conditions. We have demonstrated the fastest travel time at the $2 \mathrm{~mm}$ dash task by the proposed MagPieR robot and external field controller.

To evaluate the propulsion efficiency, highspeed video analyses were performed. They revealed the better aligning motion of the type II.1 compared to the type II.2, the horizontal trench improving the propulsion linearity with the cost of a slightly slower actuation. The redundant travel after the collision to the wall behind the goal line was observed. Since these can slow down the propulsion, we achieved the proper input impulse to make a complete stop close to the goal line.

Further improvement of the propulsion performance will be attempted with more advanced surface adhesion studies in different environments (dry and wet). Incorporating piezoelectric effect will help to overcome these unavoidable frictions. Then the ability to maneuver them should be the next challenges.

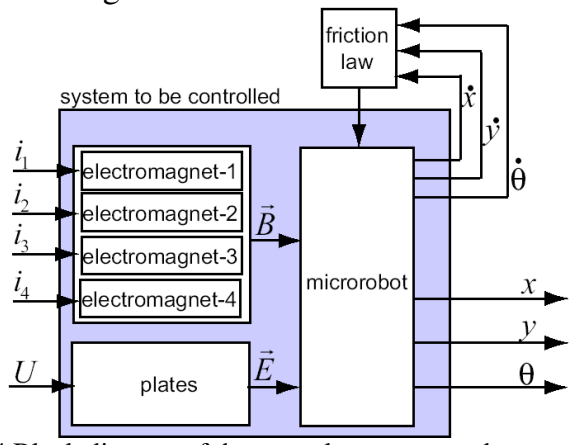

Fig.14 Block diagram of the complete system to be controlled.

In the future works, and for the 2011 Challenge, we plan to analyze the behaviour of the microrobot like a multivariable system manner (Fig.14), performing $x-y-\theta$ planar motions. Using a high sample rate reference camera and the derived behaviour analysis, we will design a closed-loop control law able to provide high performance velocity and trajectory tracking for the microrobot. The friction between the microrobot and the base is considered as disturbance since it generates unwanted stick-slip phenomenon during the movement. If it is modelled, it can be accounted inside the closed-loop control design or for a feedforward disturbance rejection. An efficient friction model that can be used is the elastoplastic model that depends on both velocity and relative displacement of the microrobot. By this way, MagPieR could be controlled in closed loop in order to achieve other challenges of the 2011 competition.

\section{REFERENCES}

[1] NIST \& IEEE Mobile Microrobotics Challenge: http://www.nist.gov/eeel/semiconductor/robots 042710_program.cfm and http://icra2010.grasp.upenn.edu/?q=RobotChallenge , Anchorage Alaska USA, May 2010.

[2] M. Sitti, "Miniature devices: voyage of the microrobots," in Nature, vol. 458, 2009, pp. 1121-1122.

[3] N. Darnton, L. Turner, K. Breuer and H. C. Berg, "Moving fluid with bacterial carpets," in Biophysical Journal, vol. 86, 2004, pp. 1863-1870.

[4] B. Behkam and M. Sitti, "Design methodology for biomimetic propulsion of miniature swimming robots," in Journal of Dynamic Systems, Measurement, and Control, vol. 128, 2006, pp. 36-43.

[5] E. B. Steager, J. Patel, C. Kim, D. Yi, W. Lee and M. Kim, "A novel method of microfabrication and manipulation of bacterial teamsters in low Reynolds number fluidic environments," in Microfluidics and Nanofluidics, vol. 5, 2008, pp. 337-346.

[6] S. Martel, M. Mohammadi, O. Felfoul, Z. Lu and P. Poupanneau, "Flagellated magnetotactic bacteria as controlled MRI-trackable propulsion and steering systems for medical nanorobots operating in the human microvasculature," in The International Journal of Robotics Research, vol. 28, 2009, pp. 571-581.

[7] B. R. Donald, C. G. Levey, C. D. McGray, I. Parpotny and D. Rus, "An untethered, electrostatic, globally controllable MEMS micro-robot," in Journal of Microelectromechanical Systems, vol. 15, 2006, pp. 1-15.

[8] Y. Yamagata, T. Higuchi, N. Nakamura and S. Hamamura, "A micro mobile mechanism using thermal expansion and its theoretical analysis," pp. 142-147.

[9] Y. Yamagata, T. Higuchi, H. Saeki and H. Ishimaru, "Ultrahigh vacuum precise positioning device utilizing rapid deformations of piezoelectric elements," in Journal of Vacuum Science and Technology, vol. 8, 1990, pp. 4089-4100.

[10] C. Pawashe, S. Floyd and M. Sitti, "Modeling of experimental characterization of an untethered magnetic micro-robot," in The International Journal of Robotics Research, vol. 28, 1009, pp. 1077-1094.

[11] D. R. Frutiger, K. Vollmers, B. E. Kratochvil and B. J. Nelson, "Small, fast, and under control: wireless resonant magnetic micro-agents," in The International Journal of Robotics Research, vol. 54, 2009, pp. 169-178.

[12] L. Zhang, J. J. Abbott, L. X. Dong, B. E. Kratochvil, D. J. Bell and B. J. Nelson, "Artificial bacterial flagella: fabrication and magnetic control," in Applied Physics Letters, vol. 94, pp. 064107.

[13] G. Hwang, S. Haliyo and S. Regnier, "Remotely powered propulsion of helical nanobelts," in Robotics: Science and Systems, 2010.

[14] M. Kummer, J. J. Abbott, B. E. Kratochvil, R. Borer, A. Sengul, B. J. Nelson, "OctoMag: An Electromagnetic System for 5-DOF Wireless Micromanipulation", Proc. of IEEE International Conference on Robotics and Automation (ICRA), May 2010.

[15] C. Pawashe, S. Floyd and M. Sitti, "Multiple magnetic microrobot control using electrostatic anchoring," in Applied Physics Letters, vol. 94, 2009, pp. 164108.

[16] A. Becker and T. Bretl, "Motion planning under bounded uncertainty using ensemble control," in Robotics: Science and Systems, 2010.

[17] M. Gauthier, S. Régnier, S. Rougeot and N. Chaillet, "Forces analysis for micromanipulations in dry and liquid media," in Journal of MicroMechatronics, vol. 3, 2006, pp. 389-413.

[18] P. Dupont, V. Hayward, B. Armstrong and F. Altpeter, "Single state elastoplastic models," in IEEE Transactions on Automatic Control, vol. 47, 2002.

[19] K. Yesin, K. Vollmers and B. Nelson, "Modeling and Control of Untethered Biomicrorobots in a Fluidic Environment Using Electromagnetic Fields," in The International Journal of Robotics Research, vol. 25, 2006, pp. 527-536. 